\title{
SLUP: A Standard List of Usability Problems in Multimodal Video Games designed for People Who Are Blind
}

\author{
Ticianne Darin \\ Instituto Universidade Virtual \\ Universidade Federal do Ceará \\ ticianne@virtual.ufc.br
}

\author{
Rossana Andrade \\ Depto. Ciência da Computação \\ Universidade Federal do Ceará \\ rossana@great.ufc.br
}

\author{
Jaime Sánchez \\ Depto. Ciência da Computação \\ Universidade do Chile \\ jsanchez@dcc.uchile.cl
}

\begin{abstract}
This paper summarizes the proposal and validation of the Standard List of Usability Problems (SLUP), a categorized list of typical problems affecting the interaction of people who are blind with audio- and haptic-based multimodal video games. SLUP can help designers to avoid recurrent usability issues in the design of multimodal games for users who are blind. Besides, evidence decurrent from user tests showed that SLUP may be further expanded and used as a ground to develop specific usability evaluation instruments.
\end{abstract}

\section{Author Keywords}

Usability evaluation; multimodal video games; audio-haptic interfaces; interaction modes; people who are blind.

\section{ACM Classification Keywords}

H.5.2. Information interfaces and presentation: User Interfaces (D2.2, H1.2, I3.6);

\section{INTRODUÇÃO}

A literatura apresenta evidências de que videogames multimodais baseados em áudio e háptica têm grande potencial para ajudar pessoas cegas a desenvolver novas habilidades [2]. No entanto, é importante destacar que o desenvolvimento e o aprimoramento destas habilidades cognitivas dependem da capacidade do jogo de traduzir informações abstratas em modalidades de interação, elementos de interface e tipos de feedback perceptíveis e significativos para pessoas cegas $[4,5]$. Consequentemente, as modalidades, controles e feedback do jogo devem ser conjugados para permitir uma interpretação precisa da informação abstrata. Caso contrário, usuários cegos poderão interpretar incorretamente os elementos e tarefas do jogo, o que os distrairia do objetivo de desenvolver habilidades.

Logo, é necessário assegurar que a interação multimodal e os elementos da interface se adequem aos propósitos cognitivos do jogo e que os jogadores possam interagir de

Permission to make digital or hard copies of all or part of this work for personal or classroom use is granted without fee provided that copies are not made or distributed for profit or commercial advantage and that copies bear this notice and the full citation on the first page. Copyrights for components of this work owned by others than the author(s) must be honored. Abstracting with credit is permitted. To copy otherwise, or republish, to post on servers or to redistribute to lists, requires prior specific permission and/or a fee. Copyright 2018 SBC.

IHC 2018, Anais Estendidos do XVII Simpósio Brasileiro sobre Fatores Humanos em Sistemas Computacionais

Outubro 22-26, 2018, Belém, Brasil

Artigos Internacionais maneira agradável e correta. Para conseguir isso, uma avaliação cuidadosa da usabilidade deve ser realizada considerando que a aplicabilidade dos Métodos de Avaliação de Usabilidade (Usability Evaluation Methods UEM) tradicionais para domínios distintos é discutível [1] e que UEM tradicionais são projetados principalmente para usuários sem deficiências.

Visando fortalecer o corpo de conhecimento sobre usabilidade e pessoas cegas, além de compartilhar resultados com a comunidade brasileira, o presente artigo resume a pesquisa originalmente publicada em [5]. Tal pesquisa apresenta duas contribuições principais para a literatura atual na área. Primeiro, a discussão detalhada dos problemas típicos que afetam a interação de pessoas cegas com jogos multimodais baseados em áudio e háptica. Os problemas de usabilidade mais comuns na interação de pessoas cegas com jogos multimodais foram organizados em uma lista (Standard List of Usability Problems - SLUP [5]), a qual visa, primeiro, ajudar designers a evitar problemas recorrentes de usabilidade durante o projeto de jogos multimodais para pessoas cegas. Segundo, evidências decorrentes de testes com usuários mostraram que SLUP pode ser expandida e usada como base para desenvolver instrumentos específicos para avaliação de usabilidade nesse contexto. Um trabalho posterior [4] - que é decorrente da pesquisa original resumida no presente artigo demonstra o uso de SLUP para criar um instrumento de usabilidade.

\section{METODOLOGIA}

Hartson et al. (2001) [6] propõem a identificação de um conjunto "padrão" de problemas de usabilidade que ocorrem em um sistema alvo, como base para comparação de UEM. Partindo deste princípio, SLUP foi estabelecida como um primeiro passo para comparar a adequação de diferentes UEM para avaliar jogos multimodais projetados para desenvolver habilidades cognitivas em pessoas cegas.

Neste trabalho, com o objetivo de tornar os resultados mais abrangentes, em vez de escolher uma aplicação-alvo, um conjunto de cinco videogames multimodais foi escolhido e analisado. Devido às diferentes combinações de dispositivos de interação e tipos feedbacks, a análise dos problemas de usabilidade nesses jogos revelou diversos tipos de problemas que ocorrem quando os usuários cegos interagem com jogos multimodais. $\mathrm{Na}$ análise dos relatórios de usabilidade dos cinco jogos, foram identificados 22 
problemas de usabilidade únicos (não duplicados) relatados pelos usuários e 35 problemas únicos apontados por avaliadores.

Para validar a lista de problemas inicialmente obtida, sete especialistas em avaliação de usabilidade e dois professores especializados em pessoas cegas responderam a um questionário de 58 itens, no qual puderam avaliar os problemas de usabilidade e explicar seu ponto de vista. $\mathrm{O}$ segundo passo para validar a lista de problemas foi a realização de sessões de avaliação de usabilidade com usuários com diagnósticos oftalmológicos diferentes (todos legalmente cegos), em ambiente real.

\section{SLUP: VISÃO GERAL}

SLUP $^{1}$ consiste em uma lista estruturada contendo 61 problemas de usabilidade relacionados à interação de pessoas cegas com jogos multimodais, ao comportamento dos usuários durante $\mathrm{o}$ jogo $\mathrm{e}$ às características dos usuários. Além dos problemas gerais de usabilidade relacionados à facilidade de aprendizado, eficiência, satisfação e dificuldades para lidar com as diferentes modalidades, SLUP detalha problemas de relacionados aos principais aspectos da interface de jogos multimodais: áudio, personalização, modo de interação e feedback [3]. Os problemas são agrupados em cinco subcategorias:

- Áudio: 15 problemas que podem afetar sons icônicos, espaciais e estéreo, além de earcons abstratos, ou síntese de fala e áudio falado;

- Personalização: 6 problemas que podem ser causados pelo tamanho, cores ou contraste de elementos gráficos, ou até mesmo pela velocidade e intensidade de sons e vozes;

- Usabilidade Geral: 13 problemas relacionados à usabilidade geral do jogo, abordando facilidade de aprendizado, satisfação, erros e eficiência;

- Feedback: 8 problemas que podem ocorrer em resposta à interação do usuário na forma de retorno háptico (cinestésico ou tátil), auditivo ou visual; e

- Interação: 19 problemas que podem estar relacionados às técnicas e dispositivos de interação e que afetam as entradas e saídas do jogo.

Dados foram coletados das sessões de teste com usuários que incluíram diferentes UEM. A evidência é que a análise da interação gravada em vídeo pode revelar a maior quantidade dos problemas de usabilidade listados na SLUP em todas as dimensões analisadas. A análise mostrou que SLUP pode ajudar a identificar, de forma eficaz e rápida, problemas gerais de usabilidade, de interação e feedback nesse contexto. Porém, a análise do vídeo é mais eficiente para revelar problemas de áudio, o que pode ser feito de maneira menos demorada se o avaliador utilizar a SLUP como heurísticas para identificar problemas.

\footnotetext{
${ }^{1}$ SLUP está disponível em http://bit.ly/SLUP-full
}

\section{CONCLUSÃO}

Neste artigo foi resumido como os problemas de usabilidade mais comuns na interação de pessoas cegas com jogos multimodais foram organizados em uma lista denominada Standard List of Usability Problems (SLUP). O objetivo de SLUP é ajudar pesquisadores e profissionais de duas maneiras. Primeiro, a evitar problemas recorrentes de usabilidade desde primeiros estágios do design desses jogos. Segundo, ajudar a identificar e corrigir problemas reais que afetam a interação multimodal nesses jogos. Finalmente, SLUP pode ser expandida pela comunidade de IHC para desenvolver novas pesquisas sobre métodos e instrumentos para avaliar a interação de pessoas cegas com diferentes tipos de interfaces.

\section{REFERÊNCIAS}

1. Parmit K. Chilana, Jacob O. Wobbrock, and Andrew J. Ko. 2010. Understanding usability practices in complex domains. In Proceedings of the SIGCHI Conference on Human Factors in Computing Systems (CHI '10). ACM, NY, USA, 2337-2346.

2. Erin C. Connors, Elizabeth R. Chrastil, Jaime Sánchez, and Lotfi B. Merabet. 2014. Action video game play and transfer of navigation and spatial cognition skills in adolescents who are blind. Frontiers in Human Neuroscience 8, 133.

3. Ticianne Darin, Jaime Sánchez, and Rossana Andrade. Dimensions for the design and evaluation of multimodal videogames for the cognition of people who are blind. 2015. In: Proceedings of the 14th Brazilian Symposium on Human Factors in Computing Systems. ACM. p.20-29.

4. Ticianne Darin, Rossana Andrade, Jaime Sánchez. 2018. CLUE: A Usability Evaluation Checklist for Multimodal Video Games Field Studies with Children Who Are Blind In: Proceedings of the 51th Annual Hawaii International Conference on Computer Science. IEEE. p. 245-254

5. Ticianne Darin, Rossana Andrade, Lotfi Merabet, and Jaime Sánchez. 2017. Investigating the Mode in Multimodal Video Games: Usability Issues for Learners who are Blind. In: Proceedings of the CHI Conference Extended Abstracts on Human Factors in Computing Systems (CHI EA '17). ACM, NY, USA, 2487-2495.

6. Rex Hartson, Terence S. Andre, and Robert C. Williges. 2003. Criteria for Evaluating Usability Evaluation Methods. International Journal of Human-Computer Interaction 15, 1, 145-181.

7. Jaime Sánchez. 2003. Software Usability for Blind Children Questionnaire (SUBC). http:// dx.doi.org/10.13140/RG.2.2.36286.08003 